\title{
EL 'CENTRO' Y LA 'PERIFERIA' DE LA CATEGORÍA SUSTANTIVO EN ESPAÑOL
}

\author{
María Tadea díaz Hormigo \\ (Universidad de Cádiz)
}

\begin{abstract}
RESUMEN
The present paper constitutes an attempt to establish a delimitation between central and peripheral elements in the category noun in Spanish according to the criterial mentioned as relevant for this distinction by the linguistics of Prague school. Two major aspects are analysed. First, the relational character of the nouns (it is relational the noun which is the nuclear element of an abstract syntactic-semantic pattern which consists of some linguistic variables). In this respect, it may be proved that is a characteristic of the spanish nouns, unlike verbs, to be no relational. Second, particular attention is paid to the nouns which have a formal-semantic relation with others units of the language system. In our opinion, the nouns which are no relational and/or which are relationed with other unit go away from the centre of this category and they gradually pass (infiltrate) into the peripheral domain of other or others categories.
\end{abstract}

1. La distinción centro / periferia fue formulada por lingüistas pertenecientes, en su mayoría, a la denominada Nueva Escuela de Praga, a partir de la tesis praguense que defiende la asimetría (no uniformidad) y carácter abierto del sistema del lenguaje, la cual supone el rechazo, por una parte, de la concepción que asegura que todos los elementos del lenguaje gozan del mismo grado de integración en el mismo ${ }^{1}, y$, por otra, de la creencia de que las categorías lingüísticas están perfectamente separadas y delimitadas en compartimentos o cajas independientes. No obstante, estos autores no llegan a establecer unas definiciones exactas y precisas de los conceptos centro y periferia, dependiendo la delimitación entre elementos centrales y periféricos, en la mayoría de los casos, del nivel o aspecto del lenguaje en el que esté basado o se haya realizado

2 Por 'grado de integración de un elemento' entienden los lingüistas praguenses «the degree of its participation in the relations characterizing the given system seen as an ensemble of its elements» (J. Vachek (1966), p. 23). 
En este sentido, hemos considerado necesario aunar las distintas posturas y criterios más significativos empleados por unos y otros, para poder así perfilar los mencionados conceptos y llevar a cabo su aplicación a la categoría sustantivo en español.

Nos manifestamos de acuerdo con lo sustentado por O.Leška(1966), para el que sólo el análisis completo y exhaustivo de la estructura lingüística permite una delimitación entre caracteres centrales y periféricos y, de acuerdo con los mismos, la posterior clasificación de los elementos en centrales, periféricos y los que ocupan posiciones intermedias entre ambos polos. Apunta que es un criterio cuantitativo el que permite diferenciar más fácilmente entre aquello que constituye o se aproxima al centro y lo que se aleja de éste.

No muy diferente es lo establecido por F. Danes (1966), quien puntualiza que es precisamente al intentar clasificar las unidades lingüísticas en categorías diferentes cuando se observa con mayor claridad que éstas no están perfectamente delimitadas ${ }^{3}$ y que es necesario perfilar previamente los caracteres centrales que definen a las diferentes clases. En este sentido, es preciso entender que cada clase o subclase de elementos está formada por un centro y una transición gradual dentro de una periferia que se introduce en la periferia de otra categoría o en las de dos o más. Por tanto, coincide con Leška al concebir la organización de los elementos del sistema de la lengua de acuerdo con el principio de CentroPeriferia-Transición, englobándose en ésta los elementos que, según Leskka, ocupan posiciones intermedias.

Indica F. Danes que para la distinción entre elementos centrales y elementos

2 Así, O. Leška (1966) apunta que existe una inclinación a relacionar el carácter «periférico» con «l'irrégularité de forme (nous disons par ex.: «les verbes irréguliers tchèques jsem, vím, jím se trouvent à la périphérie du système morphologique des verbes»), avec la non-productivité formative des mots, avec la réalisation limitée de l'opposition de signification (par ex. «l'opposition des verbes factitifs ou causatifs et des verbes simples d'action ou d'état se trouve en tchèque à la périphérie des oppositions significatives verbales») et en général avec l'exceptionnel (par ex. «à la périphérie se trouve la validité d'aspect du verbe russe vygljadet'»), avec l'éventualité, la variabilité et la rareté (par ex. «la fonction du participium perfecti pas. du verbe sans copule dans le sens passé est en russe un phénomène limité», «le passé impersonnel des verbes intransitifs kak auknetsja, tak $i$ otkliknetsja est considéré en russe comme un phénomène périphérique») etc.; de plus, nous parlons aussi de la périphérie du vocabulaire dans le cas, par ex. de l'argot» (op. cit., p. 53) y J. Popela (1966) señala que la «oposición» de centro y periferia ha sido interpretada como la que se establece entre «(1) quantitatively strong types vs. quantitatively weak types; (2) types constituted by basic features $v s$. types constituted by special features (as, e. g., nasals in the consonantal system, genitive II and locative II in the Russian system of cases); (3) «full» or «normal» types $v s$. «defective» types (cf. common names $v s$. proper names -the latter as dictionary entries have no general lexical meaning; monophthongs vs. diphthongs); (4) «pure» types vs. intermediate types; (5) productive types $v s$. unproductive types; (6) general or abstract components of the system $v s$. concrete components of the system» (op. cit., p. 71).

3 Cita F. Daněs como ejemplos representativos de la no delimitación entre categorías y subcategorías los problemas que surgen a la hora de establecer una distinción entre palabras derivadas y compuestas, grupos sintácticos y compuestos simples, y verbos copulativos frente a no copulativos. 
periféricos hay que tener en cuenta criterios cuantitativos y cualitativos, entre los que destacan la frecuencia de aparición de una determinada unidad lingüística, y, siguiendo a J. Vachek (1966), la integración y utilización (rendimiento) de una unidad en el sistema.

2. La aplicación de la dicotomía centro / periferia a la categoría sustantivo en español requiere que previamente expongamos qué entendemos por sustantivo y también, por la utilización que del mismo haremos para distinguir entre sustantivos centrales y sustantivos periféricos, el concepto de esquema sintácticosemántico con sustantivo nuclear.

Nuestro punto de vista axiomático inicial —supuesto fenomenológico de Teoría del Lenguaje y no de Lingüística General-- se basa en la aceptación de la hipótesis funcionalista que establece que el sustantivo es una clase de palabras con el significado categorial, en el sentido husserliano, 'lo que se concibe como ser en sí', un significado léxico intralingüístico, que responde al modo de ver, clasificar o dividir la realidad cada comunidad idiomática y en virtud del cual establecemos oposiciones entre los sustantivos, y, además, eventualmente, significado gramatical o auxiliar que determina la combinación de los significados categoriales y léxicos ${ }^{4}$.

Asimismo, consideramos que cualquier construcción ${ }^{5}$ con sustantivo núcleo que tenga sentido y referencia eventuales en el hablar es actualización de un esquema sintáctico-semántico suboracional subyacente que tiene como núcleo a ese sustantivo, entendiendo por esquema sintáctico-semántico con sustantivo nuclear el signo lingüístico con una forma significante (esquema sintáctico suboracional) a la que corresponde una función semántica (esquema semántico suboracional), que es abstracto ${ }^{6}$, potencial ${ }^{7}$, no predicativo ${ }^{8}$ y está constituido

4 Esta concepción de sustantivo implica, por una parte, que asumimos la formulación de E. Husserl de cuatro modos universales posibles del concebir fenomenológico: a) como existente en sí o ser en sí; b) como ser en otro o existiendo en otro; c) como modo del ser o del existir, y d) como modificación de una relación ya establecida, y, por otro lado, que admitimos la distinción de $\mathrm{E}$. Coseriu (1978) entre a) significado léxico, correspondiente, según el lingüista rumano, al «qué de la aprehensión del mundo extralingüístico, por ejemplo, el significado que es común a todas las palabras de cada una de las series: caliente - calor - calentar, rico-riqueza - enriquecer, blanco blancura - blanquear - blancamente, y que, al mismo tiempo, diferencia a cada una de estas series, como un todo, de otras series del mismo tipos (op. cit., pp. 136-137); b) significado categorial, que corresponde al «cómo de la aprehensión del mundo extralingüístico; por ejemplo, el significado que es diferente en cada caso en las palabras de la serie rico-riqueza-enriquecer» (idem, p. 137), y c) significado instrumental, que es el significado de los morfemas, con independencia de si son palabras o no, por ejemplo, $-s$ en mesa-s tiene el significado "pluralizador» (cf. ibidem).

5 Empleamos el término 'construcción', frente a 'sintagma' y 'grupo de palabras', por ser el más vacío de contenido, ya que los últimos citados parecen remitir, a tenor de su empleo en los tratados de lingüística general y las definiciones en los diccionarios de lingüística (cf. en este sentido, fundamentalmente, J. Dubois et alii (1973) y W. Welte (1985)), más específicamente a la combinación de, al menos, dos elementos, autónomos o no, y es evidente la existencia de construcciones nominales formadas sólo por un sustantivo nuclear, por ejemplo, Lo que hace falta es dinero, Libros tenemos muchos pero ninguno narra aventuras, etc. 
necesariamente por una palabra con, al menos, el significado categorial 'lo que se concibe como ser en sí' y significado léxico, $y$, en casi todos los casos, por esta palabra y una o más variables lingüísticas?.

3. Si se une los dos principios apuntados por O. Leška para distinguir entre elemento o carácter central y elemento o carácter periférico, a saber, análisis y clasificación, con el criterio cuantitativo señalado por F. Daneš, se observa que es característica de los verbos, frente a los sustantivos, adjetivos y adverbios, el ser núcleos de esquemas sintáctico-semánticos con una o más variables lingüísticas ${ }^{10}$, pues sólo algunos de los que expresan eventos y fenómenos

$6 \quad$ El esquema sintáctico-semántico con sustantivo nuclear es abstracto porque se obtiene a partir de un proceso de abstracción que consta de las siguientes fases: a) se extrae los sintagmas con sustantivos como núcleo del texto; b) se hace caso omiso de los determinantes (singularizadores o actualizadores por ser signos de individualización o de referencia); c) completamos los sintagmas defectivos con las variables lingüísticas sin las que no podría ser concebido, las cuales son las generalizaciones máximas de los sentidos y denotaciones de los sintagmas, proformas y expresiones encastradas susceptibles de determinar dinámicamente al sustantivo nuclear; d) sustituimos los sintagmas nominales dependientes también por variables, y e) eliminamos aquéllas con las que el sustantivo núcleo no contrae una relación sintáctico-semántica en el nivel subyacente, ya que sin éstas el sustantivo podría ser concebido como tal. El resultado es un esquema sintáctico suboracional completo al que corresponde un esquema semántico de su mismo nivel, que se determina por la aplicación de un inventario de pruebas semánticas del tipo ¿qué hace $X$ ?, ¿qué actitud muestra $X$ respecto a $Y$ ?, ¿qué efecto produce $X$ sobre $Y$ ?, etc. Así, por ejemplo, el esquema sintáctico subyacente al sintagma nominal el desmesurado cariño que los ancianos de la residencia sienten por los jóvenes estudiantes que van a cuidarlos los fines de semana es [cariño-de alguien-por/hacia alguien], y el esquema semántico, [actitud no activa-afectado portador de la actitud-objeto de la actitud].

Para las marcas y pruebas semánticas usadas en el establecimiento de los esquemas semánticos citados en este trabajo, cf. el apéndice.

7 Es potencial porque, aunque tiene un significado suboracional completo, carece de sentido, pues éste sólo lo adquiere en un contexto y situación comunicativa concreta, esto es, cuando se inserta en el dinamismo discursivo.

8 No se establece una relación predicativa o existencial, indicativa de un determinado modo del suceder, entre los elementos del esquema sintáctico-semántico nominal y la realidad extralingüística.

9 Por tanto, todo sustantivo, al igual que todo verbo, es, en el nivel abstracto de indagación, núcleo de un esquema sintáctico-semántico, si bien habría que distinguir entre a) sustantivos que son núcleos de esquemas sintáctico-semánticos cerovalentes, esto es, constituidos sólo por el sustantivo nuclear, por ejemplo, nieve, núcleo del esquema sintáctico-semántico [nieve : evento], subyacente al sintagma nominal la intensa nieve de los últimos días, y b) sustantivos que ocupan la posición nuclear en esquemas sintáctico-semánticos no cerovalentes, es decir, en esquemas formados por el sustantivo núcleo y una o más variables, como, por ejemplo, enfermedad, núcleo del esquema sintáctico-semántico [enfermedad : caracterización resultativa, de alguien : caracterizado], que subyace al sintagma nominal la grave enfermedad de la niña que vive en el piso de arriba.

Para otras características de los esquemas sintáctico-semánticos con sustantivos nucleares, cf. M ${ }^{2} \mathrm{~T}$. Díaz Hormigo (en prensa).

10 El esquema sintáctico-semántico con verbo en posición nuclear constituye lo que, en terminología del modelo que seguimos en nuestras investigaciones, la teoría de los esquemas sintáctico-semánticos, se denomina esquema sintáctico-semántico oracional, esto es, un «signo potencial, abstracto, completo e independiente de cualquier situación y contexto, constituido al menos por un predicado (v. g., llueve) o por un predicado y una serie de variables intralingüísticas relacionadas con él (alguien, algo, algún modo, algún lugar, algún momento)» (V. Báez San José (1987), pp. 76-77). 
metereológicos son cerovalentes ${ }^{11}$, esto es, núcleos de esquemas sintácticosemánticos constituidos por el verbo núcleo predicativo y sin ninguna variable determinadora. Esto hace que el conjunto de los verbos con esquema sintácticosemántico no cerovalente se constituya como el centro de la categoría verbal frente al conjunto de los que son núcleos de esquemas cerovalentes, que pertenecen, por tanto, a la periferia de esta categoría.

Por otra parte, puesto que la mayoría de las unidades de las clases sustantivo, adjetivo y adverbio son no relacionales ${ }^{12}$, los sustantivos, adjetivos y adverbios núcleos de esquemas sintáctico-semánticos no cerovalentes son los elementos periféricos de estas categorías e introducidos en la periferia de la categoría verbo ${ }^{13}$.

4. De este modo, el ser o no núcleo de un esquema sintáctico-semántico cerovalente, o lo que es lo mismo, el no ser o sí ser relacional, es la principal característica para la delimitación entre centro y periferia en la categoría sustantivo (sustantivos centrales y sustantivos periféricos). Ahora bien, para establecer los distintos grados de alejamiento / acercamiento al centro o a la periferia es necesario tener en cuenta otros criterios, entre los que destaca el de la posible conexión semántico-formal, en el sentido amplio del término, del sustantivo con una unidad del mismo o de distinto significado categorial ${ }^{14}$.

Así, atendiendo fundamentalmente a los dos caracteres señalados, a saber, 1) ser o no núcleo de un esquema sintáctico-semántico no cerovalente, esto es, ser o no relacional, y 2) presentar o no una conexión semántico-formal con otra unidad, hemos confeccionado la siguiente escala dentro de la categoría sustantivo en español, procediendo de la delimitación de los sustantivos más periféricos hasta llegar a los más centrales.

11 Decimos que sólo algunos porque hay un grupo de verbos de evento que son núcleos de esquemas sintáctico-semánticos monovalentes, esto es, con verbo núcleo predicativo y sólo una variable, por ejemplo, [algo : objeto no afectado, resulta : evento] y [algo : objelo no afectado, sucede : evento].

12 Denominamos relacionales a las unidades que en el esquema sintáctico-semántico implican necesaria y obligatoriamente una relación sintáctica y semántica con otra u otras variables, las cuales, en el nivel discursivo o textual, pueden ser actualizadas, investidas categorial, léxica y/o gramaticalmente y dotadas de una denotación y un sentido, o bien ser omitidas o ser designadas por alguna proforma, pero que pertenecen al esquema sintáctico-semántico en el que esa unidad aparece en posición nuclear.

13 No obstante, el número de sustantivos relacionales es mayor que el de adjetivos y el de adverbios, perteneciendo a estas últimas subclases, entre otros, los adjetivos capaz (de algo), incapaz (de algo), útil (para algo), inútil (para algo), apto/-a (para algo), llenol-a (de algo) y falto/-a (de algo), y los adverbios lejos (de algún lugar) y cerca (de algún lugar).

14 Otros aspectos que habría que examinar son, por ejemplo, los relativos a consideraciones de índole formal y semántica, $o$, ya más particularmente, la carencia de significado gramatical por parte de determinados sustantivos, como teléfono, leche, casa, paraguas, de los que decimos que son masculinos of femeninos siguiendo un criterio normativo, frente a los que sí lo tienen (perro, niño). No obstante, estos criterios servirian para delimitar sólo los sustantivos del grupo que señalaremos en último lugar, esto es, los sustantivos no relacionales no conexionados con otra unidad, los cuales, de acuerdo con los criterios seguidos en la clasificación, constituyen el centro de la categoría sustantivo. 
1) Los sustantivos relacionales conexionados formal y semánticamente con verbos y núcleos de esquemas sintáctico-semánticos suboracionales que presentan correspondencia sintáctica y semántica (el mismo número de variables lingüísticas e idéntico carácter sintáctico-semántico) con un esquema sintáctico-semántico oracional son los más cercanos al centro de la categoría verbo, $\mathrm{y}$, por tanto, los más periféricos de la categoría sustantivo.

De acuerdo con la distinción praguense centro / periferia, estos sustantivos son unidades intermedias entre las categorías sustantivo y verbo, ya que participan de características de ambas. Así, en tanto que pertenecientes a la categoría nominal, tienen el significado categorial 'lo que se concibe como ser en sí y un significado léxico, que es común (cf. nota 4) al sustantivo y al verbo núcleo predicativo del esquema sintáctico-semántico oracional con el que se relaciona el esquema sintáctico-semántico que presenta en posición nuclear a ese sustantivo, y se acercan a la categoría verbal porque en los esquemas sintáctico-semánticos suboracionales de los que son núcleos están determinados por una o más variables lingüísticas, que son las mismas, aunque no necesariamente con la misma forma, que las del esquema sintáctico-semántico oracional con el que cada esquema suboracional está relacionado.

Citamos como ejemplos los sustantivos canto y racionamiento, núcleos de los esquemas sintáctico-semánticos [canto : acción causativa, de algo : objeto no afectado, por parte de alguien: agente no afectado] y [racionamiento: acción causativa, de algo : objeto afectado, por parte de alguien: agente no afectado], que se corresponden con los esquemas oracionales [alguien: agente no afectado, canta : acción causativa, algo : objeto no afectado // algo : objeto no afectado, es cantado : proceso causativo no actitudinal agentivo, por parte de alguien: agente no afectado] y [alguien: agente no afectado, raciona: acción causativa, algo : objeto afectado // algo: objeto afectado, es racionado : proceso causativo no actitudinal agentivo, por parte de alguien: agente no afectado $]^{15}$, respectivamente.

2) Los sustantivos relacionales con conexión formal y semántica con adjetivos son núcleos de esquemas sintáctico-semánticos suboracionales que mantienen correspondencia sintáctica y semántica con esquemas oracionales en los que aparece como atributo el adjetivo relacionado con el sustantivo.

Por tanto, las unidades sustantivas de este grupo ocupan una posición intermedia entre las categorías sustantivo, adjetivo y verbo, ya que, en tanto que sustantivos, tienen el significado categorial 'lo que se concibe como ser en sî' y

15 Estos esquemas sintáctico-semánticos suboracionales presentan correspondencia semántica con el esquema oracional activo y correspondencia sintáctica con el pasivo, de ahí que hayan sido citados los dos. No obstante, el que esquema oracional activo y esquema oracional pasivo denoten o se refieran a un mismo fenómeno de la realidad no quiere decir que ambos tengan un mismo significado lingüístico, pues mientras que en el primer caso aparece obligatoriamente un agente, en el esquema pasivo es posible la eliminabilidad de esta variable (cf. al respecto V. Báez San José y M. Moreno Martínez (1985), p. 56, nota 7). 
un significado y al adjetivo que funciona como atributo en el esquema sintáctico-semántico oracional relacionado con ei esquema sintáctico-semántico que tiene como Rúclico a ese sustantivo, y participan de las características del verbo porque son núcleos de esquemas sintáctico-semánticos suboracionales constíuidos por una - más variables lingüísticas, que son las mismas, aunque no con la misma formá, que las de los esquemas sintáctico-semánticos oracionales atributivos.

Pertenecen a esto grupo los sustantivos belleza y blancura, núcleos de los esquemas sintáctico-semánticos [belleza : caracterización no restiliativa, de alguien : caracterizadol, lbelleza : caracterización resultativa, de alguien: caracterizadol, Iblancura : caracterización no resultativa, de algo : objeto caracterizadol y blancura : caracterización resultativa, de algo: objeto caracterizado], telacionados con los esquemas sintáctico-semánticos oracionales lalguien :caracterizado, es : caracterización no resultativa, bello : caracteristica], lalguien : caracierizado, está : caracterización resultativa, bello : característica], lalgo : objeio caracterizado, es: caracterización no resultativa, blanco: caracterísical y lalgo : objeto caracterizado, está : caracterización resultativa, blanco : caracterísicaj, respectivamente.

3) Los sustamtivos relacionales conexionados formal y scmánticamerie con adverbios son núclcos de esquemas sintáctico-semánticos suboracionales que se relacionar con esquemas sintáctico-semánticos oracionales en los que aparece como complemento predicativo el adverbio con el que el sustantivo presenta similitud de significado léxico, pero, además, estos sustantivos conexionados con adverbios están relacionados formal y semánticamente con adjetivos. Tienen estas características, entre otros, los sustantivos lejanía y cercanía, que intervienen en las series lejanía-lejano-lejos y cercanía-cercano-cerca, respectivamente.

Cada sustanivo do este subconjunto es, por tanto, una unidad intermedia entre las categorias sustantivo, adverbio, adjetivo y verbo, ya que, puesto que es sustantivo, tiene el significado categorial 'lo que se concibe como ser en sí y un significado léxico común a, al menos, tres unidades de significados categoriales distintos: el sustanivivo, un adjetivo y el adverbio que es complemento predicativo en el esquemé sintáctico-semántico oracional que se corresponde con el esquema sintáctico-semántico que tiene como núcleo al sustantivo, y comparte las características de la categoría verbo por ser núcleo de una serie de variables inguísticas que se corresponden, aunque no formalmente, con las del csquema sintáctico-semántico oracional en el que el adverbio desempeña una determinada función sintáctica.

Confróniense al respecto los esquemas sintáctico-semánticos de los sustantivos citados-Icercanía: caracterización resultativa, de algo :objeto caracterizado, respecio a algín lugar: adlativo real] y [lejanía : caracterización resultativa, de algo: objeto caracterizado, respecto a algún lugar : ablativo reali-, los cuales 
se relacionan, respectivamente, con los esquemas oracionales [algo : objeto caracterizado, está : caracterización resultativa, cerca : característica, de algún lugar : adlativo real] y [algo : objeto caracterizado, está : caracterización resultativa, lejos: característica, de algún lugar : ablativo real].

4) Los sustantivos relacionales con conexión formal y semántica con verbos y núcleos de esquemas sintáctico-semánticos suboracionales sin correspondencia con esquemas sintáctico-semánticos oracionales están más lejos del centro de la categoría verbo y más cerca del centro de la categoría sustantivo que los de 1). No obstante, son también unidades periféricas entre las categorías sustantivo y verbo, que se distancian del centro de la clase de palabras sustantivo y se acercan a la periferia de la categoría verbal por a) ser núcleos de esquemas sintáctico-semánticos no cerovalentes, y b) estar relacionados formal y semánticamente con un verbo, aunque no existe en este caso similitud semántica entre el sustantivo y el verbo.

Sirvan como ejemplos los sustantivos profesión y sección, relacionados formal y semánticamente con los verbos profesar y seccionar, respectivamente, y núcleos de los esquemas sintáctico-semánticos [profesión : caracterización no resultativa, de algo : objeto caracterizado] y [sección : caracterización no resultativa, de algo : objeto caracterizado].

5) Los sustantivos relacionales conexionados formal y semánticamente con un sustantivo pertenecen a la periferia de las categorías verbo y sustantivo por ser núcleos de esquemas sintáctico-semánticos no cerovalentes, si bien éstos no mantienen correspondencia con un esquema sintáctico-semántico oracional, sino con el que tiene como núcleo el sustantivo con el que aquél está conexionado. En este sentido, estas unidades están más cercanas al centro de la categoría sustantivo que las del apartado anterior.

Se clasifican en este grupo los sustantivos ahijado y madrina, núcleos de los esquemas sintácticos [ahijado : caracterización no resultativa, de alguien : caracterizado] y [madrina : caracterización no resultativa, de alguien : caracterizado], relacionados con [hijo : caracterización no resultativa, de alguien : caracterizado] y [madre : caracterización no resultativa, de alguien: caracterizado], respectivamente ${ }^{16}$.

6) Los sustantivos relacionales sin conexión con otra unidad pertenecen a la periferia de la categoría sustantivo por ser núcleos de esquemas sintáctico-

16 Hemos documentado un caso de sustantivo con conexión formal y semántica con otro sustantivo y que es núcleo de un esquema sintáctico-semántico que no mantiene relación con el esquema del que es núcleo este sustantivo sino con un esquema sintáctico-sernántico oracional. Nos referimos, concretamente, al sustantivo amistad, núcleo de los esquemas sintácticos [amistad de alguien y alguien] y [amistad de alguien con alguien], relacionados con [alguien y alguien son amigos] y [alguien es amigo de alguien], respectivamente. 
semánticos no cerovalentes, si bien éstos no presentan en ningún caso correspondencia con un esquema sintáctico-semántico que tenga como núcleo un elemento con un significado categorial igual o distinto. Así, estos sustantivos están más cerca del centro de la categoría sustantivo y más lejos del centro de la categoría verbo que los del apartado anterior.

Valgan a modo de ejemplo los sustantivos raíz y brazo, núcleos de los esquemas sintácticos [raíz de algo] y [brazo de alguien/de algo].

7) Los sustantivos no relacionales conexionados formal y semánticamente con verbos son núcleos de esquemas sintáctico-semánticos suboracionales que se corresponden con esquemas sintáctico-semánticos oracionales cerovalentes. Además, el significado léxico es común al sustantivo y al verbo con el que éste está relacionado.

El ser núcleos de esquemas sintáctico-semánticos cerovalentes los acerca al centro de la categoría sustantivo, pero la similitud semántica con el verbo los sitúa más en la periferia que los que aparecen en los apartados siguientes.

Son de este grupo los sustantivos lluvia y trueno, núcleos de los esquemas sintáctico-semánticos suboracionales [lluvia : evento] y [trueno : evento], relacionados respectivamente con los esquemas oracionales [llueve : evento] y [truena : evento].

8) Los sustantivos no relacionales con conexión formal y semántica con un verbo y que no son núcleos de esquemas sintáctico-semánticos suboracionales que se corresponden con esquemas sintáctico-semánticos oracionales no están en el centro de la categoría sustantivo sólo por estar relacionados con un verbo, si bien no existe similitud de significado léxico entre ambas unidades.

Responden a las características señaladas sustantivos como habitación y televisión, que se relacionan con los verbos habitar y televisar, respectivamente.

9) Los sustantivos no relacionales sin conexión formal y semántica con otra unidad, como mesa, perro y niño, son el centro de la categoría sustantivo.

\section{APÉNDICE: RELACIÓN ALFABÉTICA DE LAS MARCAS Y PRUEBAS SEMÁNTICAS USADAS PARA LA DETERMINACIÓN DE LOS ESQUEMAS SEMÁNTICOS}

Ablativo real: Marca semántica de la variable lingüística al gún lugar $\left(\mathrm{Y}^{17}\right)$ que responde afirmativamente a las preguntas « $¿$ Indica $Y$ separatividad locativa para que se cumpla la caracterización de $X$ ?» e « ¿Indica $Y$ lugar real?»

Acción causativa: Marca semántica del núcleo del esquema que responde a las preguntas «¿Qué hace $X$ ?» 0 «QQué hace $Y$ ?» y a «¿Qué efecto se produce

17 Los términos $\mathrm{X}$ e $\mathrm{Y}$ hacen referencia a la primera y segunda variable lingüística del esquema sintáctico-semántico oracional o suboracional. 
por la acción?»

Actitud no activa: Marca semántica del sustantivo núcleo del esquema que sí responde a la pregunta «¿Qué actitud muestra $X$ respecto a $Y$ ?» pero no a *»¿Qué hace X?»

Adlativo real: Marca semántica de la variable lingüística algún lugar (Y) que responde afirmativamente a las preguntas « ¿Indica $Y$ proximidad locativa para que se cumpla la caracterización de X?» e «¿Indica Y lugar real?»

Afectado portador de la actitud: Marca semántica de la variable lingüística alguien $(\mathrm{X})$ de la que se pueden formular las preguntas «¿Qué le pasa a $\mathrm{X}$ ?» $\mathrm{y}$ «¿Qué actitud muestra $X$ respecto a $Y$ ?»

Agente no afectado: Marca semántica de la variable alguien (X o Y) de la que se puede formular la pregunta «¿Qué hace $X$ ?» 0 «¿Qué hace $Y$ ?» pero no * ¿QQué le pasa a X?»o *»¿Qué le pasa a Y?»

Característica: Marca semántica de la variable lingüística atributo $(Y)$ de la que se puede formular la pregunta «¿Es $Y$ característica de $X$ ?»

Caracterización no resultativa: Marca semántica del núcleo del esquema

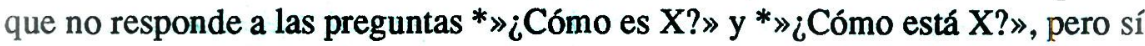
a «¿Qué característica tiene $X$ ?»

Caracterización resultativa: Marca semántica del núcleo del esquema que

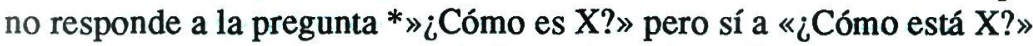

Caracterizado: Marca semántica de la variable lingüística alguien (X) de la que se puede formular la pregunta «¿Qué característica tiene X?»

Evento: Marca semántica del núcleo del esquema que no responde a las

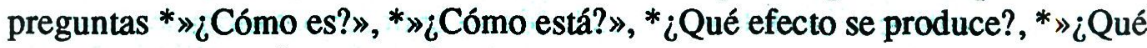
actitud se muestra?» y ${ }^{*} Q$ ué se hace?» pero sí responde a la pregunta «¿Qué pasa?»

Objeto afectado: Marca semántica de la variable lingüística algo (X o Y) de la que se puede formular la pregunta «¿Qué le pasa a X?» o «QQué le pasa a $Y$ ?»

Objeto caracterizado: Marca semántica de la variable lingüistica algo (X) de la que se puede formular la pregunta «¿Qué característica tiene X?»

Objeto de la actitud: Marca semántica de la variable lingüistica alguien que responde a la pregunta «¿Cuál es el objeto de la actitud de $\mathrm{X}$ ?»

Objeto no afectado: Marca semántica de la variable linguiística algo ( $\mathrm{X}$ o

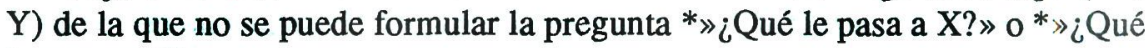
le pasa a Y?»

Proceso causativo no actitudinal agentivo: Marca semántica del núcleo predicativo de la diátesis pasiva que sí responde a las preguntas «¿Qué efecto se produce por la acción?» y «¿Qué hace Y?», pero no a *»¿Qué actitud se muestra?» 


\section{REFERENCIAS BIBLIOGRÁFICAS}

BÁEZ SAN JOSÉ, V. (1987), «Oración y esquema oracional», Lingüística Española Actual, IX, pp. 65-82.

BÁEZ SAN JOSÉ, V. Y MORENO MARTÍNEZ, M. (1985), «Funciones semánticas oracionales», Lingüística Española Actual, VII, pp. 55-85.

COSERIU, E. (1978), «Semántica y Gramática», Gramática, Semántica, Universales, Madrid, Gredos, pp. 128-147.

DANES, F. (1966), «The Relation of centre and periphery as a language universal», Travaux Linguistiques de Prague, 2, pp. 9-21.

DÍAZ HORMIGO, $M^{\mathrm{a}} \mathrm{T}$. (en prensa), «El concepto de esquema sintácticosemántico de construcción con sustantivo nuclear», Actas del I Congrés de Lingüística General (Panorama de la investigació lingüística a l'estat Espanyol), València, 1994.

DUBOIS, J., GIACOMO, M., GUESPIN, L., MARCELLESI, C., MARCELLESI, J.-B., MÉVEL, J.-P. (1973), Diccionario de Lingüística, Madrid, Alianza, 1979.

LESKĂ, O. (1966), «Le centre» et «la périphérie» des différents niveaux de la structure linguistique», Travaux Linguistiques de Prague, 2, pp. 53-57.

POPELA, J. (1966), «The functional structure of linguistic units and the system of language», Travaux Linguistiques de Prague, 2, pp. 71-80.

VACHEK, J. (1966), "On the integration of the peripherical elements into the system of language», Travaux Linguistiques de Prague, 2, pp. 23-37.

WELTE, W. (1985), Lingüística moderna. Terminología y bibliografía, Madrid, Gredos. Versión española de F. Meno Blanco. 